\title{
Oito semanas de treinamento intervalado na doença vascular periférica: um estudo de caso
}

\author{
Eight weeks of interval training on the peripheral vascular disease: a case study
}

\author{
Tiago Pereira Camposa, Rafael Bueno Orcy ${ }^{b}$, Gustavo Dias Ferreirac \\ a Fisioterapeuta. Mestre em Educação Física/Aprendizagem Motora pela Universidade Federal de Pelotas (UFPel). Pós-graduação em Fisioterapia Cardiorrespiratória \\ e Terapia Intensiva, Universidade Católica de Pelotas, Brasil. \\ b Fisioterapeuta. Doutor em Fisiologia pela Universidade Federal do Rio Grande do Sul (UFRGS). Departamento de Fisiologia, UFPel, Brasil. \\ c Fisioterapeuta. Doutor em Fisiologia pela UFRGS. Curso de Fisioterapia, Universidade Federal do Pampa, Brasil.
}

RESUMO

Introdução: O exercício intervalado é caracterizado por períodos de alta intensidade, seguido por períodos de moderada intensidade para recuperação, podendo levar a benefícios nas funções musculares e endotelial, consistindo em uma alternativa no tratamento da doença vascular periférica.

Objetivo: O objetivo deste estudo foi observar o efeito do treinamento intervalado de oito semanas na capacidade física submáxima em um paciente com doença vascular periférica.

Descrição do Caso: Foi realizado um estudo de caso com um paciente do sexo masculino, 70 anos, com índice de massa corporal dentro da normalidade $\left(24,7 \mathrm{~kg} / \mathrm{m}^{2}\right)$, fumante, sedentário e diabético (insulino-dependente), com o diagnóstico de doença vascular periférica há um ano. O estudo foi realizado durante oito semanas, com a aplicação de um treinamento intervalado de caminhada de alta intensidade e exercícios de força para os membros inferiores. Após oito semanas de treinamento o paciente diminuiu a frequência cardíaca e sensação subjetiva de cansaço no repouso, aumentou a distância no teste de caminhada de seis minutos em 24\%, suportou maior intensidade de treinamento e aumentou o escore no teste de senta e levanta.

Conclusão: O treinamento intervalado associado ao treinamento de força melhorou a capacidade aeróbia e anaeróbia do paciente, e pode ser uma boa alternativa para o tratamento da doença vascular periférica.

PALAVRAS-ChAVES: Terapia por exercício. Claudicação intermitente. Doenças vasculares. Fisioterapia. Estudo de caso.

ABSTRACT

Introduction: The interval exercise is characterized by periods of high intensity, followed by periods of moderate intensity to recover, leading to benefits in muscle and endothelial functions, therefore, being an alternative treatment for peripheral vascular disease.

Objective: This study aimed to observe the effects of eight weeks of interval exercise in the sub maximum physical capacity on a patient with peripheral vascular disease.

Case Description: A case study was performed with a male patient, seventy years old, with normal body mass index $\left(24.7 \mathrm{~kg} / \mathrm{m}^{2}\right)$, smoker, sedentary and diabetic (insulin dependent), being diagnosed with peripheral vascular disease for a year. This study was performed for eight weeks with the application of a high-intensity walking interval training and strength exercises. After eight weeks of treatment the patient decreased his resting heart rate and subjective sensation of tiredness, increased the distance in the 6-minutewalking test in $24 \%$, endured greater training intensity and increased the "sit down and get up" test score.

Conclusion: The interval training associated to strength training improved the aerobic and anaerobic capacities of the patient, and may be a good alternative in the treatment of peripheral vascular disease.

KErWORDS: Exercise therapy. Intermittent claudication. Vascular diseases. Physiotherapy. Case study. 


\section{INTRODUÇÃO}

A doença vascular periférica (DVP) é um distúrbio clínico causado por uma estenose ou oclusão em artérias dos membros superiores ou inferiores. As principais causas da DVP em pacientes com mais de 40 anos são a arterosclerose e a hipercolesterolemia ${ }^{1}$, porém o risco é ainda mais elevado em pessoas fumantes, com diabetes mellitus ou hipertensão ${ }^{2,3}$. Mais de $50 \%$ dos pacientes com DVP são assintomáticos, entretanto metade apresenta uma marcha alterada, lenta e deficiente, com o sintoma de claudicação intermitente, aliviada somente no repouso ${ }^{3}$. Estes sintomas são mais comuns nos membros inferiores, e além de dor, podem ocasionar diminuição de massa muscular ${ }^{4}$.

O exercício intervalado se caracteriza por promover períodos de alta intensidade seguido por períodos de moderada intensidade para recuperação ${ }^{5}$. Os picos de alta intensidade têm como característica a predominância de rotas metabólicas anaeróbias e a recuperação é baseada nas fontes aeróbias ${ }^{6}$, assim, é possível obter benefícios com maior rapidez comparado ao treinamento somente de intensidade moderada e de longa duração ${ }^{7}$. Em indivíduos saudáveis, é possível impor carga máxima no sistema muscular e na captação do oxigênio, oferecendo uma melhor relação tempo-eficiência ${ }^{8,9}$. O treinamento intervalado de alta intensidade induz profundas adaptações fisiológicas no músculo esquelético ${ }^{10,11}$, aumentando o conteúdo de proteínas transportadoras de ácidos graxos, permitindo uma maior oxidação ${ }^{12}$ e gasto de energia; e a quantidade de enzimas oxidativas no músculo, permitindo um máximo consumo de oxigênio ${ }^{13}$, assim melhorando o desempenho físico e condicionamento aeróbio $\left(\mathrm{VO}_{2 \text { máx }}\right)^{11,13,14,15}$.

O efeito do exercício intervalado também é investigado nas variáveis clínicas, mostrando-se um importante aliado para o tratamento de algumas doenças vasculares por apresentar melhora na função muscular e endotelial e diminuição da hipertensão. Além do exercício aeróbio, de forma geral, estar associado a um aumento de vascularização periférica ${ }^{1,16}$, esta modalidade de exercício mostra-se também um importante mecanismo de controle dos efeitos deletérios da síndrome metabólica, podendo assim, melhorar a performance na DVP ${ }^{15}$.

Assim, o objetivo deste trabalho foi observar o efeito do treinamento intervalado de oito semanas na capacidade física submáxima em um paciente com doença vascular periférica.

\section{DESCRIÇÃO DO CASO}

O paciente L.J.R., do sexo masculino, 70 anos, com índice de massa corporal dentro da normalidade $\left(24,7 \mathrm{~kg} / \mathrm{m}^{2}\right)$, fumante, sedentário e diabético (uso de $30 \mathrm{U}$ de insulina/ dia), com o diagnóstico de doença vascular periférica há um ano, foi submetido a um treinamento de caminhada de alta intensidade e exercício de força nos membros inferiores por oito semanas.

O paciente já estava em tratamento com o mesmo Fisioterapeuta por 16 meses, referindo cansaço para realizar as atividades diárias e dores nos membros inferiores durante a marcha contínua. Este trabalho é o relato de uma mudança na estratégia de tratamento, com foco no treinamento com exercício intervalado, para aumentar o trabalho muscular e gasto energético, e com intervalo para recuperação, diminuindo assim as dores que limitavam a sequência do tratamento.

Foram realizadas avaliações prévias ao treinamento, como: teste de caminhada de seis minutos (para verificar a capacidade aeróbia submáxima) e teste de senta e levanta (para verificar a capacidade anaeróbia), respectivamente, com intervalo de cinco minutos entre os testes. Os sinais clínicos do paciente (saturação periférica de oxigênio $\left(\mathrm{SapO}_{2}\right)$, frequência cardíaca ( $\left.\mathrm{FC}\right)$, pressão arterial (PA) e escala subjetiva de BORG) foram acompanhados durante o período de treinamento.

O treinamento de caminhada intervalado foi realizado por 8 semanas, com uma frequência de 3 vezes por semana, totalizando 24 sessões. Antes do início de cada sessão era verificada a frequência cardíaca em repouso, e calculada a frequência cardíaca de treino pelo método de Karvonen $(\mathrm{FC}$ de reserva $=$ FCmáx - FCrepouso, então $\mathrm{FC}$ de treino $=$ (percentual da intensidade desejada $\times$ FC de reserva) + FCrepouso). A intensidade utilizada foi de 0,9 (90\%) e a FC foi controlada com auxílio de um POLAR FS2.

Nas primeiras 4 semanas (12 sessões), o paciente foi treinado intervalando 180 segundos a $60 \%$ de sua carga máxima e 90 segundos com a frequência cardíaca de treino calculada (90\%). Este ciclo era repetido por quatro vezes a cada sessão de treinamento, totalizando 18 minutos de atividade. Nas 4 últimas semanas, o ciclo de treinamento foi alterado para 2 minutos de caminhada a $60 \%$ da máxima, intercalando com 2 minutos de $90 \%$ a intensidade máxima, repetindo o ciclo por 5 vezes, totalizando 20 minutos de atividade (Tabela 1). No final do trabalho aeróbico foi reaplicada a escala de BORG ao paciente e verificada a $\mathrm{SapO}_{2}$, PA e FC. Após de cada sessão, foram realizados exercícios de força nos segmentos dos membros inferiores de forma isotônica (utilizando bandas elástica) e isométrica (em diferentes ângulos de flexão de quadril). Após oito semanas de treinamento, o paciente foi reavaliado com os testes de caminhada de seis minutos e senta e levanta. 
Tabela 1 - Protocolo de treinamento intervalado em um paciente com doença vascular periférica.

\begin{tabular}{lc}
\hline & \multicolumn{1}{c}{ Protocolo } \\
1-4 semanas & $(3 \min 60 \%$ FCreserva $+1,5$ min 90\% FC reserva $) \times 4$ \\
$5-8$ semanas & $(2 \min 60 \%$ FCreserva $+2 \min 90 \%$ FC reserva $) \times 5$ \\
\hline
\end{tabular}

$\mathrm{Na}$ avaliação prévia, o paciente tinha os sinais clínicos estáveis, com uma PA de 120/80 mmHg, $\mathrm{SapO}_{2}$ de 98\%, FC em repouso de 83 bpm e BORG de 7 na escala subjetiva de cansaço (escala de 6 até 20). Realizou os testes de caminhada de seis minutos e senta e levanta, totalizando 395 metros e 11 repetições, respectivamente. Após a primeira sessão de treinamento a pressão se manteve estável, $125 / 78 \mathrm{mmHg}$, FC aumentou para 101 bpm e o BORG foi para 11 (ver coluna pré-treinamento na Tabela 2).

Após as 8 semanas de treinamento, o paciente foi reavaliado e os testes repetidos. Os sinais clínicos em repouso permaneceram estáveis. A PA era 120/80 mmHg, $\mathrm{SapO}_{2} 98 \%$, FCrepouso diminuiu para 78 bpm e o BORG para 6, mínimo de cansaço na escala. $O$ teste de caminhada de seis minutos foi de 491 metros, aumentando 96 metros, o que equivale a um aumento de $24,3 \%$, demonstrando uma melhor capacidade física do paciente neste teste submáximo. O teste senta e levanta aumentou para 12 repetições, demonstrando um aumento da força anaeróbia dos membros inferiores (ver coluna pós-treinamento na Tabela 2).

Tabela 2 - Avaliação de sinais clínicos e testes pré-treinamento e póstreinamento intervalado de 8 semanas em um paciente com doença vascular periférica.

\begin{tabular}{|c|c|c|}
\hline & $\begin{array}{c}\text { Pré- } \\
\text { treinamento }\end{array}$ & $\begin{array}{l}\text { Pós- } \\
\text { treinamento }\end{array}$ \\
\hline $\mathrm{PA}(\mathrm{mmHg})_{\text {trepouso }}$ & $120 / 80$ & $120 / 80$ \\
\hline $\mathrm{SapO}_{2}(\%)$ *repouso & 98 & 98 \\
\hline $\mathrm{FC}(\mathrm{bpm})$ *repouso & 83 & 78 \\
\hline BORG *repouso & 7 & 6 \\
\hline PA $(\mathrm{mmHg})$ *"pós-sessão de treino & $125 / 78$ & $120 / 80$ \\
\hline $\mathrm{SapO}_{2}(\%)$ **pós-sessão de treino & 98 & 98 \\
\hline FC (bpm) **oós-sessão de treino & 101 & 105 \\
\hline BORG "pós-sessão de treino & 11 & 12 \\
\hline Teste dos $6 \mathrm{~min}(\mathrm{~m})$ & 395 & 491 \\
\hline Teste senta e levanta (repetições) & 11 & 12 \\
\hline
\end{tabular}

* Medido previamente a sessão de treinamento. O paciente permanecia em repouso por 10 minutos na posição sentada. Pré-treinamento: mensuração antes da primeira sessão. Pós-treinamento: mensuração antes da última sessão. ${ }^{* *}$ medido imediatamente a sessão de treino. Pré-treinamento: mensuração após a primeira sessão. Pós-treinamento: mensuração após a última sessão.

Após a última sessão de treinamento, os sinais clínicos também se mantiveram estáveis, porém demonstrando que o paciente foi mais resistente a alta intensidade, com PA
120/80 mmHg, FC de 105 bpm e BORG de 12 na escala subjetiva (ver coluna pós-treinamento na Tabela 2). Outro resultado que pode estar relacionado ao treinamento foi o relato do paciente sobre a diminuição do uso de insulina de 30 U pré-treino para 26 U no período pós-treino.

Este protocolo de treinamento intervalado é de simples aplicação e se mostrou efetivo para o paciente. Seus resultados podem incentivar estudos com uma população maior, com a finalidade de padronizar uma reabilitação baseada no exercício para pacientes do DVP.

Este estudo de caso foi aprovado pelo Programa de Pós-graduação em Fisioterapia Cardiorrespiratória e Terapia Intensiva da Universidade Católica de Pelotas e o paciente assinou um Termo de Consentimento Livre e Esclarecido.

\section{DISCUSSÃO}

A doença vascular periférica é uma consequência muito prevalente no paciente diabético e causa complicações motoras, como lentidão na marcha e claudicação intermitente, causando uma menor resistência ao exercício e consequente descondicionamento ${ }^{3}$. Já é claro na literatura os benefícios do exercício para prevenção e tratamento de diversas doenças, na diabetes e na DVP esta importância é evidente na melhora da sensibilidade à insulina e aumento de angiogênese $\mathrm{e}^{1,2}$.

Revisões com meta-análise já demonstraram que o exercício diminui fatores de risco cardiovasculares, aumentando colesterol HDL, e diminuindo glicose plasmática e triglicerídeos, além de melhorar a capacidade de captação de oxigênio do organismo ${ }^{17}$. Exercícios mais intensos são descritos com benefícios mais precoces na reabilitação da doença cardiovascular ${ }^{18,19}$.

O treinamento intervalado permite uma maior intensidade de trabalho em pacientes com doenças cardiovasculares, evidenciando diversos benefícios de melhora na capacidade física e qualidade de vidaa ${ }^{20,21}$. Estudos já descrevem que o exercício intervalado gera maiores ganhos na capacidade aeróbica e na função endotelial, comparados ao exercício contínuo em portadores de doenças vasculares, porém os protocolos aplicados ainda não são padronizados 22,23 .

Este estudo de caso teve o objetivo de realizar um protocolo de treinamento intervalado de alta intensidade em um paciente de 70 anos, diabético, tabagista e sedentário, durante 8 semanas. Foram avaliados sinais clínicos antes e depois do período de treinamento apresentando alguns resultados interessantes. No repouso houve uma diminuição na frequência cardíaca e da sensação de cansaço, o que sugere que o exercício foi benéfico para melhorar função cardiovascular e qualidade de vida deste paciente. Outra 
evidência foi o aumento da resistência ao exercício, capacitando o paciente a suportar um tempo e uma intensidade maior de exercício, aumento a frequência cardíaca e a sensação de cansaço subjetiva de BORG imediatamente após o termino da atividade na última semana.

A melhora na capacidade física aeróbia ficou clara no resultado do teste de caminhada de seis minutos, onde o paciente aumentou $24,3 \%$ a distância após as 8 semanas de treinamento. Diversos estudos vêm descrevendo os benefícios do exercício em pacientes com doenças cardiovasculares, melhorando a capacidade física e pico de $\mathrm{VO}_{2}$ em pacientes com insuficiência cardíaca ${ }^{24}$, e melhorando os testes de caminhada nos pacientes com vasculopatias periféricas ${ }^{25}$.

A capacidade anaeróbia foi medida de forma indireta com o teste do senta e levanta, e também demonstrou uma melhora de uma repetição comparando o período pré e pós-exercício. Além destes dados numéricos, cabe ressaltar o depoimento do paciente que se sentiu menos cansado e mais disposto para realizar as atividades diárias após o início do protocolo, e que diminuiu a dose do medicamento de insulina de $30 \mathrm{U}$ para $26 \mathrm{U}$ após o período de treinamento. Já há revisões sistemáticas que demonstram que o treinamento físico pode melhorar escores de qualidade de vida, em questionários validados, em pacientes com claudicação intermitente ${ }^{26}$.

Este estudo de caso mostrou que o exercício intervalado associado ao treinamento de força é benéfico para capacidade física e resistência ao exercício de maior intensidade em um paciente com doença vascular periférica. Estes resultados podem motivar um ensaio clínico, com um número maior de pacientes, para avaliar o efeito do exercício intervalado e sua comparação com exercício contínuo, na capacidade física e qualidade de vida nesta população, a fim de se padronizar um tratamento mais efetivo.

\section{REFERÊNCIAS}

1. de Carvalho CC, de Sa XeritaMaux DA, Tashiro T, de Moraes SR. The effect of endurance training on the neovascularization of skeletal musculature. Acta Cir Bras. 2006;21(6):398-401. http://dx.doi. org/10.1590/S0102-86502006000600008

2. O'Neill HM. AMPK and Exercise: Glucose Uptake and Insulin Sensitivity. Diabetes Metab J. 2013;37(1):1-21. http://dx.doi. org/10.4093/dmj.2013.37.1.1

3. Garcia JU. Tratamiento percutâneo de la insuficiencia arterial crónica de los miembros inferiores. Cardiovascular Risk Factors. $2002 ; 11(4): 1-8$.

4. Bergonse FN. Evaluation of arterial circulation using the ankle/ brachial blood pressure index in patients with chronic venous ulcers. An Bras Dermatol. 2006;81(2):131-5.

5. Vogiatzis I. Interval training as an alternative modality to continuous exercise in patients with COPD. Eur Respir J. 2002;20(1):12-9. http://dx.doi.org/10.1183/09031936.02.01152001
6. Dorado C, Sanchis-Moysi J, Calbet JA. Effects of recovery mode on performance, $\mathrm{O}_{2}$ uptake, and $\mathrm{O}_{2}$ deficit during high-intensity intermittent exercise. Can J Appl Physiol. 2004;29(3):227-44. http://dx.doi.org/10.1139/h04-016

7. Talanian JL, Galloway SD, Heigenhauser GJ, Bonen A, Spriet LL. Two weeks of high-intensity aerobic interval training increases the capacity for fat oxidation during exercise in women. J Appl Physiol. 2007;102(4):1439-47. http://dx.doi.org/10.1152/japplphysiol. 01098.2006

8. Vogiatzis I, Terzis G, Nanas S, Stratakos G, Simoes DC, Georgiadou O, Zakynthinos S, Roussos C. Skeletal muscle adaptations to interval training in patients with advanced COPD. Chest. 2005; 128(6):3838-45. http://dx.doi.org/10.1378/chest.128.6.3838

9. Gillen JB, Little JP, Punthakee Z, Tarnopolsky MA, Riddell MC, Gibala MJ. Acute high-intensity interval exercise reduces the postprandial glucose response and prevalence of hyperglycaemia in patients with type 2 diabetes. Diabetes Obes Metab. 2012;14(6):575-7. http:// dx.doi.org/10.1111/j.1463-1326.2012.01564.x

10. Burgomaster KA, Hughes SC, Heigenhauser GJ, Bradwell SN, Gibala MJ. Six sessions of sprint interval training increases muscle oxidative potential and cycle endurance capacity in humans. J Appl Physiol. 2005;98(6):1985-90. http://dx.doi.org/10.1152/ japplphysiol.01095.2004

11. Little JP, Safdar A, Wilkin GP, Tarnopolsky MA, Gibala MJ. A practical model of low-volume high-intensity interval training induces mitochondrial biogenesis in human skeletal muscle: potential mechanisms. J Physiol. 2010;15(588):1011-22. http://dx.doi. org/10.1113/jphysiol.2009.181743

12. Talanian JL, Holloway GP, Snook LA, Heigenhauser GJ, Bonen A, Spriet LL. Exercise training increases sarcolemmal and mitochondrial fatty acid transport proteins in human skeletal muscle. Am J Physiol Endocrinol Metab. 2010; 299(2):E180-8. http://dx.doi.org/10.1152/ ajpendo.00073.2010

13. McKay BR, Paterson DH, Kowalchuk JM. Effect of short-term highintensity interval training vs. continuous training on $\mathrm{O} 2$ uptake kinetics, muscle deoxygenation, and exercise performance. J Appl Physiol. 2009;107(1):128-38. http://dx.doi.org/10.1152/ japplphysiol.90828.2008

14. Weber CL, Schneider DA. Increases in maximal accumulated oxygen deficit after high-intensity interval training are not gender dependent. J Appl Physiol (1985). 2002;92(5):1795-801. http:// dx.doi.org/10.1152/japplphysiol.00546.2001

15. Haram PM, Kemi OJ, Lee SJ, Bendheim MO, Al-Share QY, Waldum HL, Gilligan LJ, Koch LG, Britton SL, Najjar SM, Wisløff U. Aerobic interval training vs. continuous moderate exercise in the metabolic syndrome of rats artificially selected for low aerobic capacity. Cardiovasc Res. 2009;81(4):723-32. http://dx.doi.org/10.1093/cvr/ cvn332

16. Silva AS, Zanesco A. Physicalexercise, ß-adrenergicreceptors, and vascular response. J Vasc Bras. 2010;9:1-6.

17. Pattyn N, Cornelissen VA, Eshghi SR, Vanhees L. The effect of exercise on the cardiovascular risk factors constituting the metabolic syndrome: a meta-analysis of controlled trials. Sports Med. 2013; 43(2):121-33. http://dx.doi.org/10.1007/s40279$012-0003-z$

18. Mezzani A, Hamm LF, Jones AM, McBride PE, Moholdt T, Stone JA, Urhausen A, Williams MA. Aerobic exercise intensity assessment 
and prescription in cardiac rehabilitation: a joint position statement of the European Association for Cardiovascular Prevention and Rehabilitation, the American Association of Cardiovascular and Pulmonary Rehabilitation and the Canadian Association of Cardiac Rehabilitation. Eur J Prev Cardiol. 2013; 20:442-67. http://dx.doi. org/10.1177/2047487312460484

19. Moholdt T, Madssen E, Rognmo O, Aamot IL. The higher the better? Interval training intensity in coronary heart disease. J Sci Med Sport. 2014;17(5):506-10. http://dx.doi.org/10.1016/j.jsams. 2013.07.007

20. Moholdt T, Aamot IL, Granoien I, Gjerde L, Myklebust G, Walderhaug L, Brattbakk L, Hole T, Graven T, Stølen TO, Amundsen BH, Mølmen-Hansen HE, Støylen A, Wisløff U, Slørdahl SA. Aerobic interval training increases peak oxygen uptake more than usual care exercise training in myocardial infarction patients: a randomized controlled study. Clin Rehabil. 2012;26(1):33-44. http://dx.doi. org/10.1177/0269215511405229

21. Arena R, Myers J, Forman DE, Lavie CJ, Guazzi M. Should highintensity-aerobic interval training become the clinical standard in heart failure? Heart Fail Rev. 2013;18(1):95-105. http://dx.doi. org/10.1007/s10741-012-9333-z
22. Maiorana A. Interval training confers greater gains than continuous training in people with heart failure. J Physiother. 2012;58(3):199. http://dx.doi.org/10.1016/S1836-9553(12)70113-3

23. Jakovljevic DG, Donovan G, Nunan D, McDonagh S, Trenell MI, Grocott-Mason R, Brodie DA. The effect of aerobic versus resistance exercise training on peak cardiac power output and physical functional capacity in patients with chronic heart failure. I Int J Cardiol. 2010;145(3):526-8. http://dx.doi.org/10.1016/j. ijcard.2010.04.060

24. Belardinelli R, Georgiou D, Cianci G, Purcaro A. 10-year exercise training in chronic heart failure: a randomized controlled trial. J Am Coll Cardiol. 2012;60:1521-8. http://dx.doi.org/10.1016/j. jacc.2012.06.036

25. Locatelli EC, Pelizzari S, Scapini KB, Leguisamo CP, Silva ABD. Physical exercise in peripheral occlusive arterial disease. J Vasc Bras. 2009; 8:247-54. http://dx.doi.org/10.1590/S1677-54492009000300010

26. Spronk S, Bosch JL, Veen HF, den Hoed PT, Hunink MG. Intermittent claudication: functional capacity and quality of life after exercise training or percutaneous transluminal angioplasty--systematic review. Radiology. 2005;235(3):833-42. http://dx.doi.org/10.1148/ radiol.2353040457

Parceiros:

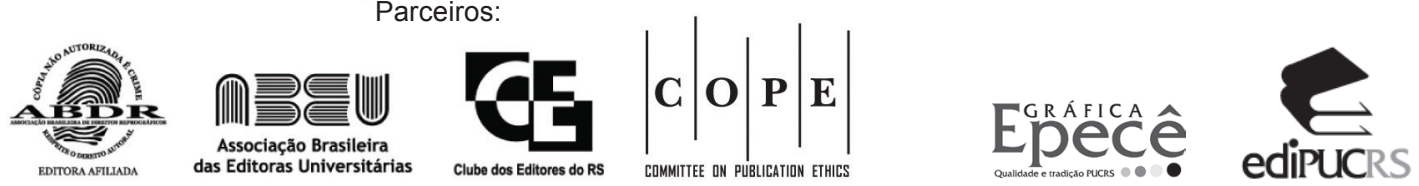

